\title{
Interactive comment on "GEM: A Dynamic Tracking Model for Mesoscale Eddies in the Ocean” by Qiu-Yang Li et al.
}

\section{Anonymous Referee \#2}

Received and published: 6 September 2016

\section{General Comments}

The work presents a new eddy tracking algorithm specifically designed to account for the dynamical processes of merging and splitting combined with a look-ahead procedure.

The topic of the Ms. is of interest and relevant since although there are already other existing algorithms for eddy detection that have been proven to be robust enough, the eddy tracking problem has not been resolved adequately. The presented tracking algorithm seems to be not only a solid method, but also treats the poorly explored dynamical processes within the evolution of the eddies (merging and splitting) in an automated way. This combined with algorithms for treating missing eddies ('eddy missing problem' -look ahead for $\mathrm{N}$ steps- ) also adds up value to the capabilities of the developed 
tracking model.

-The text is well structured and sectioned, but it has large grammatical and syntax OSD imprecisions, which have to be corrected. Also some important concepts are not well defined in a self-explanatory way (although most of them are defined in the references).

I think the subject of the manuscript is worthy of publication and interesting for the oceanic community but some Major questions have to be addressed before a second revision round and also a careful revision of the English performed.

Major Comments

\#1. There are some concepts that are used but not defined in the article (detailed in specific comments below)

\#2. I don't understand the declaration made in Section 2.2, lines 161-164 about the particles P1 and P2 in Figure 3. It is not clear what the authors refer by "particle movements", but I suppose that it should be particle trajectories of passive tracers that follow the streamlines of the geostrophic velocities derived from the SLA. In such case, the streamlines just follow the SLA contours, so that P1 and P2 follow the same trajectory (almost the same, because they are not exactly in the same SLA level) rotating around the 2 cyclones.

Geostrophic velocity definition (g gravity, $f$ coriolis parameter, $H$ SLA) $V x=-g / f d H / d y$ $\mathrm{Vy}=\mathrm{g} / \mathrm{f} d \mathrm{H} / \mathrm{dx}$

Instead, it seems that you are considering that SLA is a potential of the velocity, $V=$ $\operatorname{grad}(\mathrm{H})$, like they were falling by gravity...

\#3. There is missing the definition of the watershed (also pointed below).

\#4. As terminology, I would consider births and deaths within the dynamic processes, next to merging and splitting (and as opposite to kinematics). It's just a suggestion, and it's on you to do the changes.

Interactive

comment

Printer-friendly version

Discussion paper 
\#5. I'm not sure that criteria 1-4 for eddy identification is what define eddies. It is clear that this defines well the maximum but regarding the "territory" it is defined by the points inside the outmost SLA contour (as you state in line 141). So, you can have points around a maximum satisfying the conditions but being outside the defined boundary.

Interactive

\#6. I think the algorithm on Similarity Vector with classification T0-T3 is very smart, but comment I am wondering that the variables $r 1$ and $r 2$ defined as fraction of overlap areas would just be valid for small time steps. I know you are using AVISO product with daily time step, but did the method work for weekly data? (note that $r 1$ ( $r 2$ ) in this case would be too close to 0 (small overlaps). In this sense, I think the territory overlap may be too sensitive to the time-step, and maybe another variable (shape criteria from Mason et al. (2014) + Area, for example) could work too, being less sensitive to time-step.

\#7. Related with the previous question: in the Look-ahead, isn't the threshold rc reduced? I suppose that for $\mathrm{N}=1$ or 2 can work with no changes, but for greater N's the ratios r1 r2 will diminish because eddy movements.

\section{Specific Comments}

\#8. Concepts that would be desirable to be defined or clarified (somewhere) in the article âĂć Mononuclear / multinuclear eddy. I know what you are referring about, but it would be desirable to define them. âĂć Eddy segmentation. âĂć Watershed. How is it defined?

\#10 Page 3. L93 If the algorithms work well... What do you mean with this?

\#9. Page 4. L127 The SLA value of the eddy is above (below) a given SLA threshold How is defined the SLA value of the eddy? The SLA of the extremum? Also, you should give the threshold value (for sake of coherence... you are giving the threshold values that appear in conditions 3 and 4 in the next two lines).

Printer-friendly version

\#10. Some (not all) grammatical issues: - look ahead/ look-ahead (sometimes 
with dash/sometimes without it) - straight-line / straight line model (sometimes with dash/sometimes without it) - some names are given with small letters -e.g. multiple hyOSD pothesis assignment (MHA)- and other in capital letters -e.g. Genealogical Evolution Model- . Check it for coherence

Page 1. L 32 Such transports has $->$ have

Page 1. L 37 Eddies can also be identified from velocity fields.

Interactive

comment

Page 3. L97 The data and eddy detection method $->$ methods (... are. ..)

Page 3. L98-99 computation complex -> complexity?

Page3. L99 illuminated -> shown

Page 4. L100 data noises -> data noise

Page 4. L105 SLA is an altimetry field, not a flow field

Page 4 L106 daily -> daily (not in cursive)

Page 4. L107-108 by the AVISO -> from AVISO

Page 4. L116-117 might affect to the eddy detection

Page 4. L120 from SLA data

Page 5. L155 with a given threshold... threshold of what? You previously defined several thresholds

Page 5. L160-164 I don't understand why do you talk about particles here, and in this way...

Page 6 L175 (also L190 and others) previously-identified -> previously identified

Page 7 L203 days -> day

And the list goes on. There are many other grammatical issues that should be cor- 
rected. I am not a native English speaker but the present version of the Ms. needs to be improved.

OSD

Interactive comment on Ocean Sci. Discuss., doi:10.5194/os-2016-49, 2016.

Interactive

comment 